\title{
The Old and the New
}

A topic often debated is older methods of practice versus the more contemporary approaches. Proponents of the former argue that their approach alone is the correct one, being in strict adherence to Hahnemann's own method and even a slight deviation is a deviation from the fundamentals of Homeopathy. On the other hand, some of those in favor of the newer theories and techniques seem to rely entirely on these, with little knowledge of traditional sources.

Change is synonymous with evolution, and if Homeopathy is to consistently prove efficacious, we cannot be complacent. In fact, we should all be working jointly towards filling the lacunae in our system. At the same time change cannot be so bizarre that the original essence of a thing is lost. Any change, however immense, must be based on solid, unchangeable fundamentals. If we keep this in mind we will not be afraid to advance from the older methods of practice, and yet we will not be entirely abandoning these older methods in embracing newer ones.

The newer theories, as for example the kingdom classification, or the additions to Hahnemann's trio of miasms and new provings have been the target of some criticism. At a glance these concepts may seem to be a paradigm shift from Hahnemannian philosophy. If one examines these concepts in depth, however, one realizes that they are founded very strongly on the known materia medica and repertory.

The classification into kingdoms and subkingdoms and discovering the common feature of a particular subkingdom, family of plant or a row of the periodic table, is only an abstraction of the knowledge of the remedies we know through provings and clinical cases. By inductive logic we can apply the idea to the rest of the remedies in the family which are not so well proven. It is a kind of Grand Generalization (from Boenninghausen) applied to families of remedies.

Many masters (e.g. Clarke, Farrington, Dhawale) have been drawn to this idea. It is such an eye opener to study spiders as a group or Anacardiaceae as a group etc. And to explore the periodic table is fascinating when it is done by using what we already know through provings and discovering a pattern in the rows and columns. All this naturally takes us towards the study of the sources and a vast goldmine of information is ready to be integrated into the homeopathic treasury, if used diligently.

The knowledge of the kingdom classification helps the prescriber understand in depth some characteristic symptoms of remedies, so much so that a direct connection of the symptom to the source of the remedy is sometimes discernable. The restless nature of Tarentula and other remedies of the spider group can actually be seen in the behavior of these little animals; the rubric, Destructiveness, cunning, often used in cases of children needing this remedy, can actually be appreciated in the manner in which the spider traps and kills its prey. Often the connection of the source and the symptoms is more subtle.

It requires a careful study of the symptoms in the materia medica, and also a study of the source. The knowledge of only the symptoms may be limiting in several cases and by also studying the source one may be able to prescribe remedies with less than complete provings. On the other hand, using the knowledge of the sources without adequate familiarity with the materia medica is a recipe for disaster.

The newer approaches are never meant to substitute the traditional method of studying characteristic symptoms of the materia medica or rubrics in the repertory, but are meant to complement it. In practice one can see the two come together beautifully. The symptoms and the systematization are two sides of the same coin. The symptoms from the provings help us create the system of groups and the system helps us perceive individual remedies of the group.

To be firmly rooted in the old and ever open to the new ensures that one is not limited by the old and at the same time not blindly swayed by the new. It is important to see the new as an extension of the old, as a continuum.

Special thanks go to Munjal Thakkar for gathering many of the articles present in this issue of Links.

Rajan Sankaran, guest-editor 